

\title{
Accurate Overlap Area Detection Using a Histogram and Multiple Closest Points
}

\author{
Yonghuai $\mathrm{Liu}^{1}$, Ralph R. Martin ${ }^{2}$, Longzhuang $\mathrm{Li}^{3}$, and Baogang $\mathrm{Wei}^{4}$ \\ 1 Department of Computer Science \\ Aberystwyth University, Ceredigion SY23 3DB, UK \\ 2 School of Computer Science \& Informatics \\ Cardiff University, Cardiff, CF24 3AA, UK \\ 3 Department of Computing Science \\ Texas A and M University, Corpus Christi, TX 78412-5824, USA \\ ${ }^{4}$ College of Computer Science \\ Zhejiang University, Hangzhou 710072, P.R. China
}

\begin{abstract}
In this paper, we propose a novel ICP variant that uses a histogram in conjunction with multiple closest points to detect the overlap area between range images geing registered. Tentative correspondences sharing similar distances are normally all within, or all outside, the overlap area. Thus, the overlap area can be detected in a bin by bin batch manner using a histogram. Using multiple closest points is likely to enlarge the distance difference for tentative correspondences in the histogram, and pull together the images being registered, facilitating the overlap area detection. Our experimental results based on real range images show that the performance of our proposed algorithm enhances the state of the art.
\end{abstract}

\section{Introduction}

Range image registration finds numerous applications in areas such as 3D object modelling and recognition, computer graphics, virtual reality, reverse engineering, and industrial inspection. It has thus attracted considerable attention in the $3 \mathrm{D}$ vision community. Research into range image registration techniques has been driven by the development of 3D laser scanning technologies which provide easy 3D data acquisition, and the computational power of modern computers (see Figure 1).

Registration has two goals (i) to establish point correspondences between overlapping range images, and (ii) to estimate the rigid transformation that brings one range image into the best possible alignment with the other. The fact that these two problems must be solved simultaneously complicates the range image registration process.

\section{$1.1 \quad$ Previous work}

Due to the challenging nature of automatic range image registration, a large number of algorithms have been developed. Many are based on the iterative 

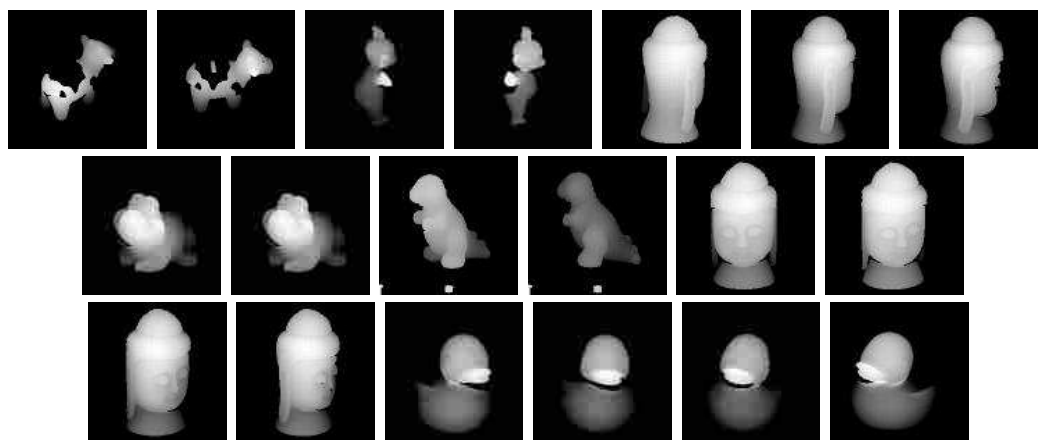

Fig. 1. Real range images used. Top, left to right: cow60 and 57, tubby 100 and 60 , buddha 140 , 120, and 100; Middle: frog20 and 0, dinosaur36 and 0, buddha0 and 20; Bottom: buddha40 and 60, duck120, 100, 80 and 60 .

closest point (ICP) approach [3], and improved variants $[1,9,11,15,16]$, as well as other approaches such as feature extraction and matching $[6,14]$, salient point detection and matching [4], density functions [21], genetic search [12, 20], scatter search [17], visibility classification and modeling [19], M-estimators [2], exhaustive search with limited points [13], graduated assignment [7], and expectation maximisation $[5,8]$; many others also exist.

These algorithms belong to three main categories, or a combination of them, depending on how the correspondences are established: (i) feature extraction and matching $[6,14]$; (ii) coarse matching of points in one image to those in another $[5,7]$, and (iii) transformation search and evaluation $[17,20]$. Each category has advantages and disadvantages:

- The first class of algorithms can establish correspondences between any two overlapping range images subject with either small or large transformations, while the second and third require that the transformation is approximately known;

- The first class of algorithms has to extract and match geometric and/or optical features from a structured, a non-structured, or an analytic representation of the surface of interest, while the second and third do not have to perform feature extraction and matching;

- Extraction of features is typically sensitive to noise caused by sampling and properties of the scanning process, and furthermore, matching of features is not straightforward because: (a) features should ideally be viewpoint invariant, (b) the similarity metric must allow discrimination of different features; and (c) a feature in one image may match multiple candidate features in another, leading to a combinatorial correspondence problem. The second class of algorithms heavily depends on both optimization and explicit outlier treatment. In contrast, while the third class typically has the advantage of finding the globally optimal solution, they are usually time consuming and it is difficult to determine appropriate termination conditions.

In summary, automatic registration of overlapping range images is still not a fully solved problem. 


\subsection{Our approach}

The ICP algorithm is a de facto standard technique for registration of overlapping free form shapes for three main reasons: (i) it is not just an algorithm, but also a methodology, which is widely used for object recognition and data clustering; (ii) it is theoretically guaranteed to establish high quality tentative correspondences [10]; and (iii) it is usually employed to refine the registration results obtained [12]. However, it introduces false correspondences in almost every iteration caused by inaccurate transformation parameters, occlusion, and appearance and disappearance of point, and so it has attracted intensive attention for improvement from the 3D vision community.

The FICP algorithm [15] is mathematically elegant, since it simultaneously estimates both the overlapping region and the registration parameters by optimizing the root mean squared distance (RMSD) of the tentative correspondences established using the traditional closest point criterion (CPC). Inspired by the FICP algorithm, in this paper, we propose a novel ICP variant that uses a histogram in conjunction with multiple closest points to determine the overlap area between the range images being registered. If certain correspondences have similar distances, typically, they will all simultaneously fall either inside or outside the overlap area. Thus, detection of the overlap area may be performed by considering batches. To implement this idea, we employ a histogram of the squared distances (SDs) between tentative correspondences: all tentative correspondences with similar distances fall into the same bin. The objective function that minimizes the Euclidean distances between the tentative correspondences is evaluated for each bin, allowing detection of the overlap area to be done in a batch manner: if any correspondence from a bin lies in the overlap area, then all the others in the same bin also lie in the overlap area and vice versa.

The SDs of the tentative correspondences determine their distribution in the histogram. To enlarge the difference between distances belonging to overlap and non-overlap areas, we replace the estimate the SD of a tentative correspondence by the sum of the SDs between a point in one image and a number of closest points in theother. In the overlap area, such Multiple Closest Points (MCPs) will not make much difference in the sense of distinguishing between different correspondences. However, in the non-overlap area, they may cause tentative correspondences to differ significantly in their SDs and thus fall into different bins of the histogram, facilitating the differentiation of real correspondences from false ones. Real correspondences will typically have lower distances.

To evaluate our proposed algorithm, we have also implemented the FICP algorithm [15] and one of the latest ICP variants, Geometric Primitive ICP (GPICP) [1]. A comparative study was carried out using real range images from a Minolta Vivid 700 range camera.

The rest of this paper is structured as follows: Section 2 details our novel algorithm, Section 3 presents experimental results, and Section 4 draws some conclusions. 

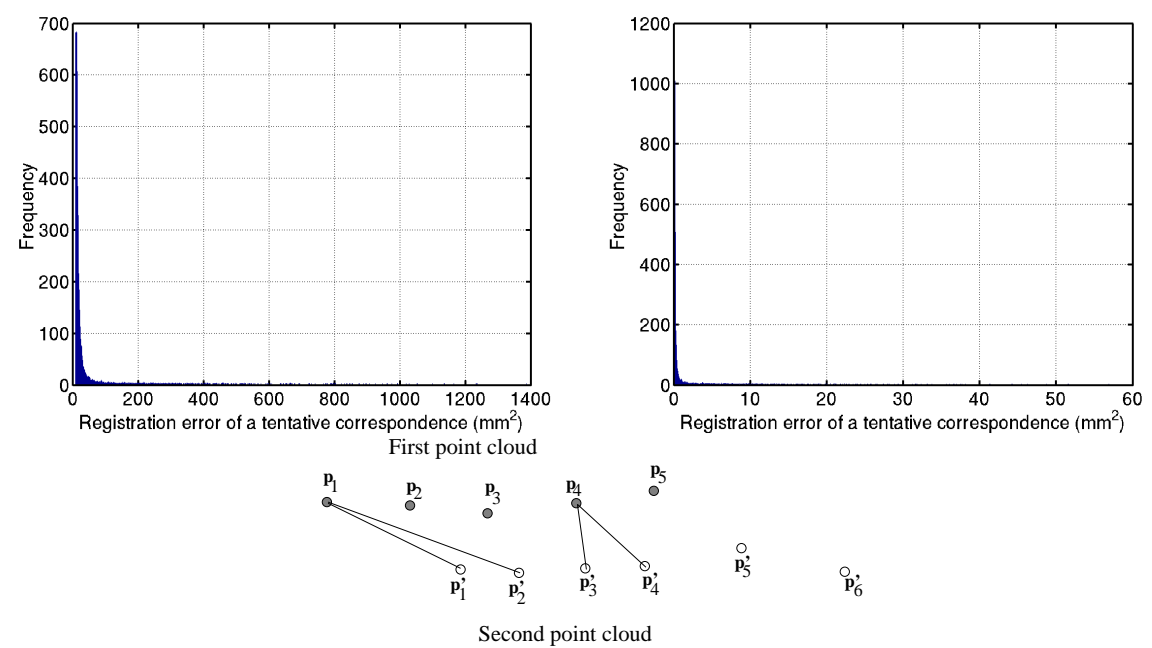

Fig. 2. Top: The histogram of SDs of tentative correspondences defined using 16 closest points (left) and a single closest point (right) respectively for the duck80 and duck60 images. Bottom: $\mathbf{p}_{1}^{\prime}$ and $\mathbf{p}_{2}^{\prime}, \mathbf{p}_{3}^{\prime}$ and $\mathbf{p}_{4}^{\prime}$ are two closest points to $\mathbf{R} \mathbf{p}_{1}+\mathbf{t}$ and $\mathbf{R} \mathbf{p}_{4}+\mathbf{t}$ respectively. The difference between $\left\|\mathbf{p}_{1}^{\prime}-\mathbf{R} \mathbf{p}_{1}-\mathbf{t}\right\|^{2}+\left\|\mathbf{p}_{2}^{\prime}-\mathbf{R} \mathbf{p}_{1}-\mathbf{t}\right\|^{2}$ and $\left\|\mathbf{p}_{3}^{\prime}-\mathbf{R} \mathbf{p}_{4}-\mathbf{t}\right\|\left\|^{2}+\right\| \mathbf{p}_{4}^{\prime}-\mathbf{R} \mathbf{p}_{4}-\mathbf{t} \|^{2}$ can be as large as twice that between $\left\|\mathbf{p}_{1}^{\prime}-\mathbf{R} \mathbf{p}_{1}-\mathbf{t}\right\|^{2}$ and $\left\|\mathbf{p}_{3}^{\prime}-\mathbf{R} \mathbf{p}_{4}-\mathbf{t}\right\|^{2}$.

\section{Algorithm}

Assume that two range images to be registered are represented as two sets of unorganised points $\mathbf{P}=\left\{\mathbf{p}_{1}, \cdots, \mathbf{p}_{n_{1}}\right\}$ and $\mathbf{P}^{\prime}=\left\{\mathbf{p}_{1}^{\prime}, \cdots, \mathbf{p}_{n_{2}}^{\prime}\right\}$, representing the same free form shape viewed under a small transformation, such that $\mathbf{P}$ and $\mathbf{P}^{\prime}$ have a relatively large overlap in $3 \mathrm{D}$ space and the pure translation motion derived from the difference of the centroids of $\mathbf{P}$ and $\mathbf{P}^{\prime}$ provides a good initial estimate for the transformation, which comprises a rotation matrix $\mathbf{R}$ and translation vector $\mathbf{t}$. Using the traditional ICP criterion [3], a set of tentative correspondences $\left(\mathbf{p}_{i}, \mathbf{p}_{c(i)}^{\prime}\right)$ is obtained between $\mathbf{P}$ and $\mathbf{P}^{\prime}$ :

$$
\mathbf{p}_{c(i)}^{\prime}=\operatorname{argmin}_{\mathbf{p}^{\prime} \in \mathbf{P}^{\prime}}\left\|\mathbf{p}^{\prime}-\mathbf{R} \mathbf{p}_{i}-\mathbf{t}\right\|
$$

where $c(i) \in\left[1, n_{2}\right]$ is the label of a point in $\mathbf{P}^{\prime}$ : this is a mapping associating $\mathbf{p}_{c(i)}^{\prime}$ in $\mathbf{P}^{\prime}$ with $\mathbf{p}_{i}$ in $\mathbf{P}$. The search for a tentative correspondent is determined by the size of $\mathbf{P}^{\prime}$. In order to speed up the search for the closest points $\mathbf{p}_{c(i)}^{\prime}$, an optimised $k$-D tree data structure [9], and squared Euclidean distance, rather than Euclidean distance itself, are employed in our implementation.

\subsection{Using a histogram to determine overlap}

Having obtained a set of tentative correspondences $\left(\mathbf{p}_{i}, \mathbf{p}_{c(i)}^{\prime}\right)$ between $\mathbf{P}$ and $\mathbf{P}^{\prime}$, the following objective function is used [15] to estimate the rotation matrix 
$\mathbf{R}$ and translation vector $\mathbf{t}$, and the fractional overlap size $f$ (meaning that $f n_{1}$ points belong to the overlap region):

$$
\operatorname{FRMSD}\left(\mathbf{P}, \mathbf{P}^{\prime}, \mathbf{R}, \mathbf{t}\right)=\frac{1}{f^{\lambda}} \sqrt{\frac{1}{\left|\mathbf{P}_{f}\right|} \sum_{\mathbf{p}_{i} \in \mathbf{P}_{f}}\left\|\mathbf{p}_{c(i)}^{\prime}-\mathbf{R} \mathbf{p}_{i}-\mathbf{t}\right\|^{2}}
$$

where $\mathbf{P}_{f}$ denotes the $f n_{1}$ points $\mathbf{p}_{i} \in \mathbf{P}$ with smallest distances $\left\|\mathbf{p}_{c(i)}^{\prime}-\mathbf{R} \mathbf{p}_{i}-\mathbf{t}\right\|$. We set $\lambda=3$ during the iterative process and $\lambda=0.95$ in a final iteration.

Suppose that certain correspondences have similar distances. Typically, they will either all be inside or all be outside the overlap area (Figure 2). Thus, deciding whether particular correspondences belong to the overlap area can be more effectively determined in batches, which we implement using a histogram.

All tentative correspondences falling in the same bin $j$ have similar SDs $d_{i}$ and are presumed to all be inside or all outside the overlap area. The SDs $d_{i}$ can be approximated as $(j+1) h_{n}^{*}$ and $j=0, \cdots, s-1$ where $s$ is the number of bins and $h_{n}^{*}$ is the bin width. The larger the parameter $s$, the better the approximation. Using this approximation, the objective function in Equation 2 can be rewritten as:

$$
\operatorname{FrmSD}\left(\mathbf{P}, \mathbf{P}^{\prime}, \mathbf{R}, \mathbf{t}, f\right)=\frac{1}{f^{\lambda}} \sqrt{\frac{1}{\left|\mathbf{P}_{f}\right|} \sum_{\mathbf{p} \in \mathbf{P}_{f}}(j+1) h_{n}^{*}}
$$

which counts the number $h_{j}$ of tentative correspondences whose SDs can be approximated by $(j+1) h_{n}^{*}$. This is exactly the idea of a histogram. As $h_{n}^{*}$ is a constant, the above objective function can be further rewritten as:

$$
\operatorname{FRMSD}\left(\mathbf{P}, \mathbf{P}^{\prime}, \mathbf{R}, \mathbf{t}, f\right)=\frac{1}{\left(\sum_{j=0}^{f} h_{j} / n_{1}\right)^{\lambda}} \sqrt{\frac{\sum_{j=0}^{f} h_{j}(j+1)}{\sum_{j=0}^{f} h_{j}}}
$$

which calculates the square root of a weighted average of the bin indexes with weights defined as the frequencies $h_{j}$ of the corresponding bins $j$.

In practice, we implement the histogram based detection of the overlap area as follows:

- Use Scott's method [18] to determine the bin width $h_{n}^{*}$ of the histogram:

$$
h_{n}^{*}=\alpha d_{\sigma} / n_{1}^{1 / 3}
$$

where $\alpha$ is a positive number that reflects the characteristics of the actual data and $d_{\sigma}$ is the standard deviation of $d_{i}=\left\|\mathbf{p}_{c(i)}^{\prime}-\mathbf{R} \mathbf{p}_{i}-\mathbf{t}\right\|^{2}$.

- Determine the number $s$ of bins in the histogram: $s=\left(d_{\max }-d_{\min }\right) / h_{n}^{*}$ where $d_{\max }=\max _{k} d_{k}$ and $d_{\min }=\min _{k} d_{k}$.

- Construct the histogram $\mathbf{H}=\left\{h_{0}, h_{1} \cdots, h_{s-1}\right\}$ of SDs $d_{i}$ of all tentative correspondences where $h_{j}$ is the frequency of $(j+1) h_{n}^{*}: h_{j} \leftarrow h_{j}+1, j=$ $\left.\left\lfloor\left(d_{i}-d_{\min }\right) / h_{n}^{*}\right)\right\rfloor$ and $i=1, \cdots, n_{1}$. 
- Compute values $J_{f}$ of the objective function in Equation 3 by changing the number $f$ of the bins in the overlap area: $J_{f}=\operatorname{FRMSD}\left(\mathbf{P}, \mathbf{P}^{\prime}, \mathbf{R}, \mathbf{t}, f\right)$, $f=0, \cdots, s-1$.

- Select the minimum $J_{f}$ to determine the points $\left(\mathbf{p}_{i}, \mathbf{p}_{c(i)}^{\prime}\right)$ in the overlap area.

Note that the computation of $J_{f}$ does not involve the actual SDs $d_{i}$ of the tentative correspondences. This is because they are approximated by $(j+1) h_{n}^{*}$ where $h_{n}^{*}$ is a constant. This property provides a novel perspective for detection of overlap area.

\subsection{Using multiple closest points to reduce error}

Earlier, the SD $d_{i}$ of a tentative correspondence $\left(\mathbf{p}_{i}, \mathbf{p}_{c(i)}^{\prime}\right)$ was defined as $d_{i}=$ $\left\|\mathbf{p}_{c(i)}^{\prime}-\mathbf{R} \mathbf{p}_{i}-\mathbf{t}\right\|^{2}$. Since $d_{i}$ plays a crucial role in determining whether or not $\left(\mathbf{p}_{i}, \mathbf{p}_{c(i)}^{\prime}\right)$ lies in the overlap area and hence in camera motion re-estimation, its exact form is important. Here, we define it as the sum of the SDs between $\mathbf{R} \mathbf{p}_{i}+\mathbf{t}$ and a number $m$ of the closest points in $\mathbf{P}^{\prime}$ (Figure 2).

Computation of $d_{i}$ can be easily implemented using a $k$-D tree without requiring significantly more computational time. Multiple closest points (MCPs) have a potential to play two roles: (i) using MCPs will not make much difference from using the single closest point in the overlap area between $\mathbf{P}$ and $\mathbf{P}^{\prime}$. However, in the non-overlap area, it is likely to enlarge the SDs of incorrect correspondences, thus making detection of the overlap area more robust. Figure 2 shows that the maximum of the SDs of the tentative correspondences defined over $16 \mathrm{MCPs}$ has been increased by as much as $(1400-60 \times 16) /(60 \times 16) \times 100 \%=45.83 \%$, manifested by the fact that the histogram is more representative of the distribution of the SDs of the tentative correspondences, and the bins of the histogram are more scattered; and (ii) they tend to pull together the images being registered and thus maximize the overlap area.

After the overlap area has been detected, the transformation parameters are estimated using the quaternion least square method [3]. When the difference of the average SD of the overlapping correspondences between two successive iterations falls below a threshold (0.000001) or the number of iterations has exceeded 300, iteration terminates. Since the proposed algorithm is a novel ICP variant based on the Histogram and Multiple closest points, it is denoted HMICP in the rest of this paper. It has a computational complexity of $O(n \log n)$.

\section{Experimental results}

To validate the performance of the proposed HM-ICP algorithm, the FICP algorithm [15] and a recent ICP variant, geometric primitive ICP (GP-ICP) [1] were also implemented and compared using a Pentium IV, 2.80GHz computer. These three algorithms employ different strategies for the detection of the overlap area: (i) sorting $d_{i}$ in the FICP algorithm takes time $O(n \log n)$, whereas constructing 

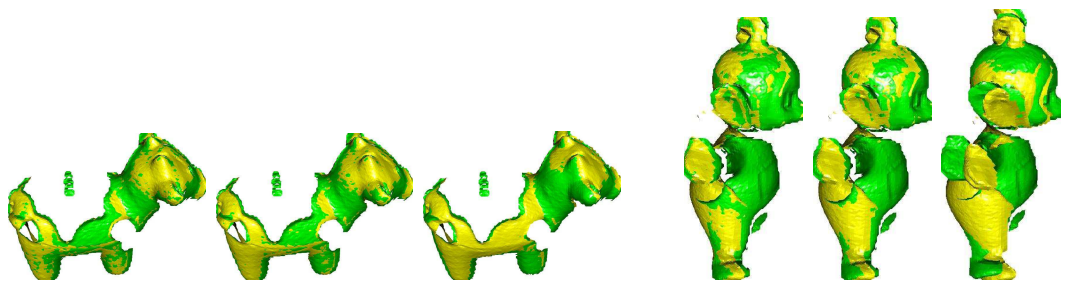

Fig. 3. Registration results for the proposed HM-ICP algorithm using two different pairs of range images and varying $\alpha$. Left three: cow60, cow57; Right three: tubby 100 , tubby60. In each case: $\alpha=0.01, \alpha=0.1, \alpha=1$.

Table 1. The mean $e_{\mu}$ and standard deviation $e_{\delta}$ of registration errors in millimetres for reciprocal correspondences (RCs), known and estimated rotation angles $\theta$ and $\hat{\theta}$ in degrees of the trasnformation, the number $N$ of RCs, and registration time $t$ in seconds for our HM-ICP algorithm with varying $\alpha$.

\begin{tabular}{|c|c|c|c|c|c|c|c|}
\hline Images & $\alpha$ & $e_{\mu}(\mathrm{mm})$ & $e_{\delta}(\mathrm{mm})$ & $\theta\left(^{\circ}\right)$ & $\hat{\theta}\left(^{\circ}\right)$ & $N$ & $t$ (sec.) \\
\hline \multirow{3}{*}{ cow60-57 } & 0.01 & 0.49 & 0.19 & \multirow{3}{*}{30} & 30.39 & 3249 & 9 \\
\hline & 0.10 & 0.50 & 0.19 & & 30.17 & 3255 & $\overline{7}$ \\
\hline & 1.00 & 0.71 & 0.29 & & 28.80 & 3215 & 5 \\
\hline \multirow{3}{*}{ tubby $100-60$} & 0.01 & 0.29 & 0.19 & \multirow{3}{*}{40} & 39.13 & 2056 & 20 \\
\hline & 0.10 & 0.29 & 0.19 & & 37.96 & 2053 & 17 \\
\hline & 1.00 & 0.52 & 0.33 & & 27.31 & 1649 & 10 \\
\hline
\end{tabular}

a histogram has a computational complexity of $O(n)$; (ii) the FICP algorithm detects the overlap area by considering each correspondence in turn, whereas the histogram determines the overlap area by considering bins, and there are far fewer bins than points; (iii) the FICP algorithm uses the same objective function for the detection of the overlap area and the estimation of the trasnformation, while our HM-ICP algorithm separates them; and (iv) while both HM-ICP and FICP algorithms simultaneously optimize the registration parameters and the overlap area, the GP-ICP algorithm employs thresholds for the rejection of false correspondences and minimizes the point to point distance at early stages of registration and the point to plane distance at later stages.

The comparative study used real range images (Figure 1) downloaded from a publicly available database hosted at the Signal Analysis and Machine Perception laboratory at Ohio State University. They were captured using a Minolta Vivid 700 range camera, and are all of size of $200 \times 200$ pixels. The purpose of these experiments is threefold: (i) to determine how to set parameters such as the histogram bin width and the number $m$ of closest points in our HM-ICP algorithm, (ii) to test whether the histogram and MCPs can effectively detect the overlap area, and (iii) whether our HM-ICP algorithm advances the state of the art. 

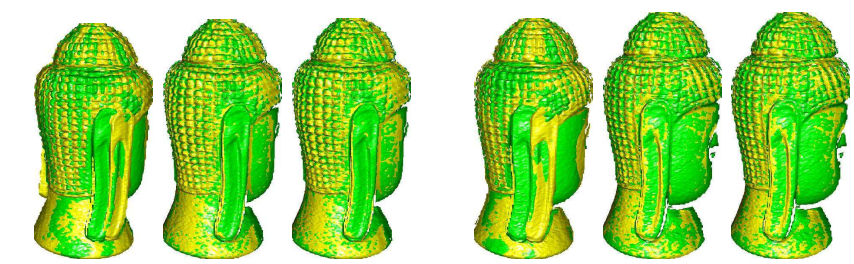

Fig. 4. Registration results for our HM-ICP algorithm applied to different overlapping range images using different values of $m$. Left three: buddha140, buddha120; Right three: buddha120, buddha100. In each case: $m=1, m=16, m=25$.

Table 2. Average $e_{\mu}$ and standard deviation $e_{\delta}$ of registration errors in millimetres for RCs, known and estimated rotation angles $\theta$ and $\hat{\theta}$ in degrees for the transformation, the number $N$ of RCs, and registration time $t$ in seconds for our HM-ICP algorithm, using different numbers $m$ of closest points.

\begin{tabular}{|c|c|c|c|c|c|c|c|}
\hline Images & $m$ & $e_{\mu}(\mathrm{mm})$ & $e_{\delta}(\mathrm{mm})$ & $\theta\left(^{\circ}\right)$ & $\hat{\theta}\left({ }^{\circ}\right)$ & $N$ & (sec.) \\
\hline \multirow{3}{*}{ buddha140-120 } & 1 & 0.82 & 0.39 & \multirow{3}{*}{20} & 4.42 & 8153 & 58 \\
\hline & 16 & 0.59 & 0.25 & & 20.08 & 10070 & 163 \\
\hline & 25 & 0.59 & 0.26 & & 20.21 & 10097 & 199 \\
\hline \multirow{3}{*}{ buddha120-100 } & 1 & 0.84 & 0.45 & & 2.75 & 8061 & 30 \\
\hline & 16 & 0.58 & 0.26 & & 20.46 & 9760 & 140 \\
\hline & 25 & 0.58 & 0.26 & & 20.46 & 9755 & 152 \\
\hline
\end{tabular}

To compare the algorithms, we used the average and standard deviation in millimetres of registration errors of reciprocal correspondences (RCs), the estimated rotation angle in degrees of the transformation relative to the ground truth value, and the time taken in seconds for registration. A reciprocal correspondence $\left(\mathbf{p}_{i}, \mathbf{p}_{c(i)}^{\prime}\right)$ is one for which $i=c(c(i))$, implying that if $\mathbf{p}_{i}$ in $\mathbf{P}$ finds $\mathbf{p}_{c(i)}^{\prime}$ in $\mathbf{P}^{\prime}$ as a correspondent, $\mathbf{p}_{c(i)}^{\prime}$ in $\mathbf{P}^{\prime}$ also finds $\mathbf{p}_{i}$ in $\mathbf{P}$ as a correspondent. RCs are often used to measure the performance of registration algorithms as their determination does not involve any threshold $[1,16]$ and hence unwanted bias. In Figures 3-5, yellow represents the transformed first image $\mathbf{P}$, and green represents the second image $\mathbf{P}^{\prime}$.

\subsection{Histogram bin width}

The SD histogram bin width plays a key role in determining registration errors and speed. We investigated choice of the bin width by letting $\alpha=0.01, \alpha=0.1$, and $\alpha=1$ in Equation 4; we used the cow60, cow57 and tubby100, tubby60 images from Figure 1 for the experiments. Results are presented in Figure 3 and Table 1, and show that using a large $\alpha=1$ and hence a large bin width provides only a coarse approximation of the SDs of the tentative correspondences. While this leads to efficient registration, it gives poor registration results: e.g. the two hands of the tubby in the two images are separated. Using a small bin width, setting $\alpha=0.01$, provides a good approximation of the SDs, leading to more 

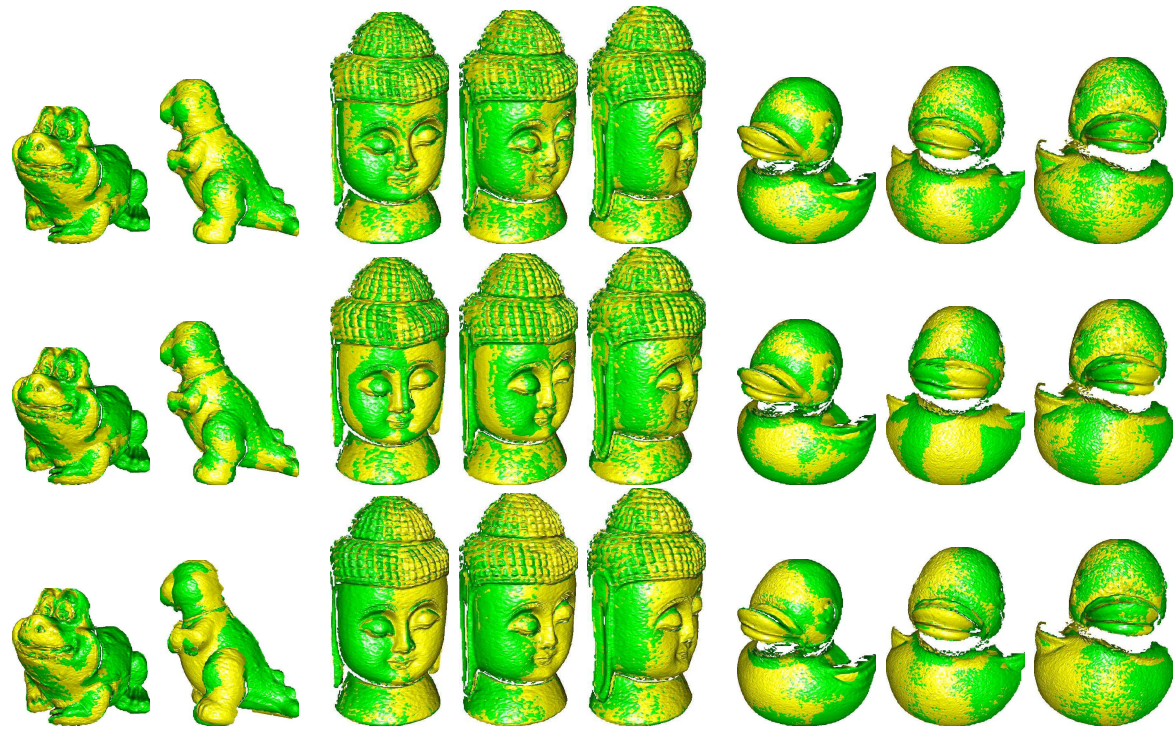

Fig. 5. Registration results for different algorithms and different range images. Top: HM-ICP; Middle: FICP; Bottom: GP-ICP. Columns from left to right: frog20-0, dinosaur36-0, buddha0-20, 20-40, and 40-60; duck80-60, 100-80, and 120-100.

accurate registration results at a cost of increased computational time. $\alpha=0.1$ provides a good compromise between accuracy and speed. Thus, in later tests, we set $\alpha=0.1$.

\subsection{Number of closest points}

The number of the closest points has a subtle effect on determination of SDs for the tentative correspondences. We experimentally investigated the effect of varying the number $m$ of closest points, setting $m=1, m=16$, and $m=25$. We used the buddha140, buddha120, and buddha120, buddha100 image pairs in Figure 1 for the experiments. Results are presented in Figure 4 and Table 2, which show that as expected, a large number of closest points, $m=25$, leads to most accurate registration results but at a cost of greater computational time, while setting $m=1$ increases computational efficiency but produces poorer results: e.g. the two ears of the buddha in the two images are misplaced. In practice $m=16$ seems a good compromise between accuracy and speed. Thus, henceforth, we set $m=16$.

\subsection{Comparative study}

In this section, we compare our new HM-ICP algorithm with FICP and GP-ICP algorithms using 8 pairs of real overlapping range images shown in Figure 1: frog20-0, dinosaur36-0, buddha0-20, 20-40, and 40-60, duck80-60, 100-80, and 
Table 3. Average $e_{\mu}$ and standard deviation $e_{\delta}$ registration errors in millimetres for RCs, known and estimated rotation angles $\theta$ and $\hat{\theta}$ in degrees of the transformation, the number $N$ of RCs, and registration time $t$ in seconds for different algorithms.

\begin{tabular}{|c|c|c|c|c|c|c|c|}
\hline Images & Algorithm & $e_{\mu}(\mathrm{mm})$ & $\mid e_{\delta}(\mathrm{mm})$ & $\theta\left(^{\circ}\right)$ & $\hat{\theta \theta}\left(^{\circ}\right)$ & $N$ & $t$ (sec.) \\
\hline \multirow{3}{*}{ frog $20-0$} & HM-ICP & 0.30 & 0.15 & \multirow{3}{*}{20} & 18.84 & 5806 & 38 \\
\hline & FICP & 0.30 & 0.15 & & 18.83 & 5798 & 16 \\
\hline & GP-ICP & 0.31 & 0.15 & & 18.58 & 5726 & 16 \\
\hline \multirow{3}{*}{ dinosaur36-0 } & HM-ICP & 0.60 & 0.54 & \multirow{3}{*}{36} & 35.11 & 5302 & 29 \\
\hline & FICP & 0.56 & 0.54 & & 35.66 & 5309 & 23 \\
\hline & GP-ICP & 1.38 & 1.41 & & 23.96 & 3321 & 31 \\
\hline \multirow{3}{*}{ buddha0-20 } & HM-ICP & 0.58 & 0.24 & \multirow{3}{*}{20} & 20.10 & 11011 & 137 \\
\hline & FICP & 0.81 & 0.49 & & 13.77 & 9353 & 64 \\
\hline & GP-ICP & 0.73 & 0.32 & & 18.18 & 10531 & 29 \\
\hline \multirow{3}{*}{ buddha20-40 } & HM-ICP & 0.58 & 0.25 & \multirow{3}{*}{20} & 20.11 & 10786 & 130 \\
\hline & FICP & 0.71 & 0.35 & & 17.16 & 9859 & 58 \\
\hline & GP-ICP & 0.67 & 0.27 & & 18.78 & 10576 & 29 \\
\hline \multirow{3}{*}{ buddha $40-60$} & HM-ICP & 0.58 & 0.25 & \multirow{3}{*}{20} & 19.81 & 10551 & 115 \\
\hline & FICP & 0.58 & 0.25 & & 19.41 & 10535 & 51 \\
\hline & GP-ICP & 0.61 & 0.25 & & 19.01 & 10472 & 26 \\
\hline \multirow{3}{*}{ duck80-60 } & HM-ICP & 0.30 & 0.17 & \multirow{3}{*}{20} & 19.08 & 7529 & 87 \\
\hline & FICP & 0.31 & 0.18 & & 17.77 & 7517 & 38 \\
\hline & GP-ICP & 0.32 & 0.17 & & 19.39 & 7454 & 23 \\
\hline \multirow{3}{*}{ duck100-80 } & HM-ICP & 0.27 & 0.15 & \multirow{3}{*}{20} & 18.06 & 7229 & 103 \\
\hline & FICP & 0.41 & 0.27 & & 6.03 & 6700 & 22 \\
\hline & GP-ICP & 0.28 & 0.14 & & 18.23 & 7144 & 25 \\
\hline \multirow{3}{*}{ duck120-100 } & HM-ICP & 0.27 & 0.12 & \multirow{3}{*}{20} & 19.19 & 7567 & 84 \\
\hline & FICP & 0.30 & 0.15 & & 16.47 & 7446 & 38 \\
\hline & GP-ICP & 0.28 & 0.12 & & 18.39 & 7551 & 27 \\
\hline
\end{tabular}

120-100. The buddha head is of a generally cylindrical shape. The duck head and belly are of a generally spherical shape. Such shapes are challenging to register, since simplicity leads to ambiguity in transformation parameters. Rresults are presented in Figure 5 and Table 3. Both the HM-ICP and FICP algorithms produced similar results for the registration of geometrically complex frog20-0 and dinosaur36-0 images. The FICP algorithm had difficulties registering the buddha and duck images, giving poor average registration errors for reciprocal correspondences and rotation angle, because (i) a point difference of the objective function in Equation 2 does not always reflect a difference in overlap area, especially when the tentative correspondences have similar distances; and (ii) an objective function suitable for the transformation estimation is not necessarily also suitable for detection of the overlap area. Our HM-ICP algorithm produces much better results, decreasing the average registration error by up to $17 \%$. As the FICP algorithm is a refinement algorithm, this error decrease is valuable and represents a significant achievement. Accurate registration results are manifested as a large amount of interpenetration [20] between the superimposed images. 
The reasons why multiple points in the same bin of the histogram are a powerful tool for the detection of the overlap area can be explained as follows. MCPs render the cause the data in the bins in the histogram to become more scattered, as seen in Figure 2. This facilitates the discrimination of correct correspondences from false ones. Tentative correspondences in the same scattered bins also play a role in perturbing the existing registration parameters and thus preventing our HM-ICP algorithm from converging prematurely. This perturbation arises due to use of MCPs about the local geometry in the images and relies on accuracy of the existing registration parameters. This is desirable and in sharp contrast with the ad hoc random approach [11] that is usually not reliable. Our HM-ICP algorithm requires an additional computational time of $33 \%$ compared to the FICP algorithm. This is because the latter usually converges prematurely.

Even though the dinosaur36-0 images are geometrically complex, the GP-ICP algorithm still produced much worse results, with average errors compared to our HM-ICP algorithm as much as 130\% higher. It exhibits a similar behaviour for registering the geometrically simple buddha and duck images. In this case, it increases the average error by as much as $12 \%$. The GP-ICP algorithm produces worse results because it is difficult to set up thresholds for the rejection of false correspondences. In the later stages of registration, it also has to invert a matrix to estimate of the registration parameters. When this matrix is close to singular, the estimated registration parameters are unreliable and may lead the GP-ICP algorithm to fail to find any true correspondence in the next iteration. On the other hand, it minimizes the point-to-plane distance, but not the point-to-point distance.

\section{Conclusions}

This paper has proposed a novel ICP variant that uses both a histogram and multiple closest points to detect the overlap area during range image registration. Our experimental results show that accurate detection of the overlap area is obtained for various real range images even with relatively simple geometry that is typically more challenging to register. This is because the role of multiple points in the same scattered bin of the histogram is more pronounced than a single point in the FICP algorithm [15] in perturbing the existing registration parameters and helping our HM-ICP algorithm to traverse the local minimum of the objective function during registration of overlapping range images. Our HMICP algorithm also outperforms the latest ICP variant, Geometric Primitive ICP (GP-ICP) [1], because our approach simultaneously optimizes both the overlap area and the registration parameters, while the latter has difficulty in choosing thresholds for rejection of false correspondences and in minimizing the point-to-

plane distance. Thus, our HM-ICP algorithm advances the state of the art for registration of overlapping range images. Future research will consider how to to reduce the computational cost of the HM-ICP algorithm. 


\section{References}

1. Bae K.-H.: Evaluation of the convergence region of an automated registration method for 3D laser scanner point clouds. Sensors 9, 355-375 (2009)

2. Banno A., Masuda T., Oishi T., Ikeuchi K.: Flying laser range sensor for large-scale site-modeling and its application in Bayon digital archival project. IJCV 78, 207-222 (2008)

3. Besl P.J., McKay N.D.: A method for registration of 3D shapes. IEEE Trans. PAMI 14, 239-256 (1992)

4. Castellani U., Cristani M., Fantoni S. and Murino V.: Sparse points matching by combining 3D mesh saliency with statistical descriptors. Computer Graphics Forum 27, 643-652 (2008)

5. Dewaele G., Devernay F., and Horaud H.: Hand motion from 3D point trajectories and a smooth surface model. Proc. ECCV, pp. 495-507 (2004)

6. Dinh H.Q., Kropac S.: Multi-resolution spin images. Proc. CVPR, pp. 863-870 (2006)

7. Gold S., Rangarajan A., et al.: New algorithms for 2-D and 3-D point matching: pose estimation and correspondence. Pattern Recognition 31, 1019-1031 (1998)

8. Granger S. and Pennec X.: Multi-scale EMICP: a fact and robust approach for surface registration. Proc. ECCV, pp. 418-432 (2002)

9. Liu Y.: Replicator dynamics in the iterative process for accurate range image matching. IJCV 83, 30-56 (2009)

10. Liu Y.: Constraints for Closest Point Finding. Pattern Recognition Letters 29, 841-851 (2008)

11. Liu Y: Improving ICP with Easy Implementation for Free Form Surface Matching. Pattern Recognition 37, 211-226 (2004)

12. Lomonosov E., Chetverikov D., and Ekart A.: Pre-registration of arbitrarily oriented 3D surfaces using a genetic algorithm. Pattern Recognition Letters 27, 12011208 (2006)

13. Marquez M.R.G., Wu S.-T.: Using simplified meshes for crude registration of two partially overlapping range images. WSCG, pp. 183-190 (2007)

14. Masuda T.: Log-polar height maps for multiple range image registration. Computer Vision and Image Understanding 113, 1158-1169 (2009)

15. Phillips J.M., Liu R., Tomasi C.: Outlier Robust ICP for Minimizing Fractional RMSD. Proc. 3DIM, pp. 427-434 (2007)

16. Pulli K.: Multiview registration for large data sets. Proc. 3DIM, pp. 160-168 (1999)

17. Santamaria J., Cordon O., Damas S., Aleman I., Botella M.: A scatter search-based technique for pair-wise $3 \mathrm{D}$ range image registration in forensic anthropology. Soft Computing 11, 819-828 (2007)

18. Scott D.W.: On optimal and data-based histograms. Biometrika 66, 605-610 (1979)

19. Sharp G.C., Lee S.W., and Wehe D.K.: Maximum-likelihood registration of range images with missing data. IEEE Trans. PAMI 30, 120-130 (2008)

20. Silva L., Bellon O.R.P., and Boyer K.L.: Precision range image registration using a robust surface interpenetration measure and enhanced genetic algorithms. IEEE Trans. PAMI 27, 762-776 (2005)

21. Wang F., et al.: Groupwise point pattern registration using a novel CDF-based Jensen-Shannon Divergence. Proc. CVPR, pp. 1283-1288 (2006) 